\title{
Sharp bounds for Seiffert and \\ Neuman-Sándor means in terms of generalized logarithmic means
}

Yu-Ming Chu ${ }^{1 *}$, Bo-Yong Long², Wei-Ming Gong ${ }^{1}$ and Ying-Qing Song ${ }^{1}$

\section{"Correspondence:}

chuyuming2005@yahoo.com.cn

'School of Mathematics and

Computation Sciences, Hunan City

University, Yiyang, 413000, China

Full list of author information is

available at the end of the article

\begin{abstract}
In this paper, we prove three sharp inequalities as follows: $P(a, b)>L_{2}(a, b)$, $T(a, b)>L_{5}(a, b)$ and $M(a, b)>L_{4}(a, b)$ for all $a, b>0$ with $a \neq b$. Here, $L_{r}(a, b), M(a, b)$, $P(a, b)$ and $T(a, b)$ are the rth generalized logarithmic, Neuman-Sándor, first and second Seiffert means of $a$ and $b$, respectively.

MSC: $26 \mathrm{E} 60$
\end{abstract}

Keywords: generalized logarithmic mean; Neuman-Sándor mean; first Seiffert mean; second Seiffert mean

\section{Introduction}

The Neuman-Sándor mean $M(a, b)$ [1] and the first and second Seiffert means $P(a, b)$ [2] and $T(a, b)[3]$ of two positive numbers $a$ and $b$ are defined by

$$
\begin{aligned}
& M(a, b)= \begin{cases}\frac{a-b}{2 \operatorname{arcsinh}\left(\frac{a-b}{a+b}\right)}, & a \neq b, \\
a, & a=b,\end{cases} \\
& P(a, b)= \begin{cases}\frac{a-b}{4 \arctan (\sqrt{a / b})-\pi}, & a \neq b, \\
a, & a=b\end{cases}
\end{aligned}
$$

and

$$
T(a, b)= \begin{cases}\frac{a-b}{2 \arctan \left(\frac{a-b}{a+b}\right)}, & a \neq b, \\ a, & a=b,\end{cases}
$$

respectively.

Recently, these means, $M, P$ and $T$, have been the subject of intensive research. In particular, many remarkable inequalities for $M, P$ and $T$ can be found in the literature $[1,4-8]$.

The power mean $M_{p}(a, b)$ of order $r$ of two positive numbers $a$ and $b$ is defined by

$$
M_{p}(a, b)= \begin{cases}\left(\frac{a^{p}+b^{p}}{2}\right)^{1 / p}, & p \neq 0, \\ \sqrt{a b}, & p=0 .\end{cases}
$$

(o 2013 Chu et al.; licensee Springer. This is an Open Access article distributed under the terms of the Creative Commons Attribution License (http://creativecommons.org/licenses/by/2.0), which permits unrestricted use, distribution, and reproduction in any medium, provided the original work is properly cited. 
The main properties for $M_{p}(a, b)$ are given in [9]. In particular, the function $p \mapsto M_{p}(a, b)$ $(a \neq b)$ is continuous and strictly increasing on $\mathbb{R}$.

The arithmetic-geometric mean $A G(a, b)$ of two positive numbers $a$ and $b$ is defined as the common limit of sequences $\left\{a_{n}\right\}$ and $\left\{b_{n}\right\}$, which are given by

$$
a_{0}=a, \quad b_{0}=b, \quad a_{n+1}=\frac{a_{n}+b_{n}}{2}, \quad b_{n+1}=\sqrt{a_{n} b_{n}} .
$$

Let $H(a, b)=2 a b /(a+b), G(a, b)=\sqrt{a b}, L(a, b)=(b-a) /(\log b-\log a), I(a, b)=$ $1 / e\left(b^{b} / a^{a}\right)^{1 /(b-a)}, A(a, b)=(a+b) / 2$, and $S(a, b)=\sqrt{\left(a^{2}+b^{2}\right) / 2}$ be the harmonic, geometric, logarithmic, identric, arithmetic and root-square means of two positive numbers $a$ and $b$ with $a \neq b$, respectively. Then it is well known that the inequalities

$$
\begin{aligned}
H(a, b) & =M_{-1}(a, b)<G(a, b)=M_{0}(a, b)<L(a, b)<A G(a, b)<I(a, b) \\
& <A(a, b)=M_{1}(a, b)<M(a, b)<T(a, b)<S(a, b)=M_{2}(a, b)
\end{aligned}
$$

hold for all $a, b>0$ with $a \neq b$.

For $r>0$ the $r$ th generalized logarithmic mean $L_{r}(a, b)$ of two positive numbers $a$ and $b$ is defined by

$$
L_{r}(a, b)=L^{1 / r}\left(a^{r}, b^{r}\right)= \begin{cases}{\left[\frac{b^{r}-a^{r}}{r(\log b-\log a)}\right]^{1 / r},} & a \neq b, \\ a, & a=b .\end{cases}
$$

It is not difficult to verify that $L_{r}(a, b)$ is continuous and strictly increasing with respect to $r \in(0,+\infty)$ for fixed $a, b>0$ with $a \neq b$.

In $[2,3]$ Seiffert proved that the double inequalities

$$
L(a, b)<P(a, b)<I(a, b)
$$

and

$$
A(a, b)<T(a, b)<S(a, b)
$$

hold for all $a, b>0$ with $a \neq b$.

The following bounds for the first Seiffert mean $P(a, b)$ in terms of power mean were presented by Jagers in [10]:

$$
M_{1 / 2}<P(a, b)<M_{2 / 3}(a, b)
$$

for all $a, b>0$ with $a \neq b$.

Hästö [11, 12] proved that the function $x \rightarrow T(1, x) / M_{p}(1, x)$ is increasing on $(0,+\infty)$ if $p \leq 1$ and found the sharp lower power mean bound for the Seiffert mean $P(a, b)$ as follows:

$$
P(a, b)>M_{\log 2 / \log \pi}(a, b)
$$

for all $a, b>0$ with $a \neq b$. 
In [13] the authors presented the following best possible Lehmer mean bounds for the Seiffert means $P(a, b)$ and $T(a, b)$ :

$$
\bar{L}_{-1 / 6}(a, b)<P(a, b)<\bar{L}_{0}(a, b) \text { and } \quad \bar{L}_{0}(a, b)<T(a, b)<\bar{L}_{1 / 3}(a, b)
$$

for all $a, b>0$ with $a \neq b$. Here, $\bar{L}_{p}(a, b)=\left(a^{p+1}+b^{p+1}\right) /\left(a^{p}+b^{p}\right)$ is the Lehmer mean of $a$ and $b$.

In $[14,15]$ the authors proved that the inequalities

$$
\begin{aligned}
& \alpha_{1} S(a, b)+\left(1-\alpha_{1}\right) A(a, b)<T(a, b)<\beta_{1} S(a, b)+\left(1-\beta_{1}\right) A(a, b), \\
& S^{\alpha_{2}}(a, b) A^{1-\alpha_{2}}(a, b)<T(a, b)<S^{\beta_{2}}(a, b) A^{1-\beta_{2}}(a, b)
\end{aligned}
$$

and

$$
\alpha_{3} T(a, b)+\left(1-\alpha_{3}\right) G(a, b)<A(a, b)<\beta_{3} T(a, b)+\left(1-\beta_{3}\right) G(a, b)
$$

hold for all $a, b>0$ with $a \neq b$ if and only if $\alpha_{1} \leq(4-\pi) /[(\sqrt{2}-1) \pi], \beta_{1} \geq 2 / 3, \alpha_{2} \leq 2 / 3$, $\beta_{2} \geq 4-2 \log \pi / \log 2, \alpha_{3} \leq 3 / 5$ and $\beta_{3} \geq \pi / 4$.

For all $a, b>0$ with $a \neq b$, the following inequalities can be found in [16, 17]:

$$
L(a, b)=L_{1}(a, b)<A G(a, b)<L_{3 / 2}(a, b)<M_{1 / 2}(a, b) .
$$

Neuman and Sándor [1] established that

$$
P(a, b)<A(a, b)<M(a, b)<T(a, b)
$$

and

$$
\frac{\pi}{2} P(a, b)>A(a, b)>\operatorname{arcsinh}(1) M(a, b)>\frac{\pi}{4} T(a, b)
$$

for all $a, b>0$ with $a \neq b$. In particular, the Ky Fan inequalities

$$
\frac{G(a, b)}{G\left(a^{\prime}, b^{\prime}\right)}<\frac{L(a, b)}{L\left(a^{\prime}, b^{\prime}\right)}<\frac{P(a, b)}{P\left(a^{\prime}, b^{\prime}\right)}<\frac{A(a, b)}{A\left(a^{\prime}, b^{\prime}\right)}<\frac{M(a, b)}{M\left(a^{\prime}, b^{\prime}\right)}<\frac{T(a, b)}{T\left(a^{\prime}, b^{\prime}\right)}
$$

hold for all $0<a, b \leq 1 / 2$ with $a \neq b, a^{\prime}=1-a$ and $b^{\prime}=1-b$.

It is the aim of this paper to find the best possible generalized logarithmic mean bounds for the Neuman-Sándor and Seiffert means.

\section{Lemmas}

In order to establish our main results, we need three lemmas, which we present in this section.

Lemma 2.1 The inequality

$$
\left(\frac{x^{4}-1}{4 \log x}\right)^{1 / 2}<\frac{x^{2}-1}{4 \arctan x-\pi}
$$

holds for all $x>1$. 
Proof Let

$$
f(x)=\frac{x^{4}-1}{4 \log x}-\left(\frac{x^{2}-1}{4 \arctan x-\pi}\right)^{2} .
$$

Then $f(x)$ can be rewritten as

$$
f(x)=\frac{\left(x^{4}-1\right) f_{1}(x)}{4(4 \arctan x-\pi)^{2} \log x},
$$

where

$$
f_{1}(x)=(4 \arctan x-\pi)^{2}-\frac{4\left(x^{2}-1\right) \log x}{x^{2}+1} .
$$

Simple computations lead to

$$
\begin{aligned}
& f_{1}(1)=0, \\
& f_{1}^{\prime}(x)=\frac{4}{1+x^{2}} f_{2}(x),
\end{aligned}
$$

where

$$
\begin{aligned}
& f_{2}(x)=8 \arctan x-\frac{4 x \log x}{x^{2}+1}-x+\frac{1}{x}-2 \pi, \\
& f_{2}(1)=0, \\
& f_{2}^{\prime}(x)=\frac{f_{3}(x)}{\left(x^{2}+1\right)^{2}},
\end{aligned}
$$

where

$$
\begin{aligned}
& f_{3}(x)=4\left(x^{2}-1\right) \log x-x^{4}+x^{2}+1-x^{-2}, \\
& f_{3}(1)=0, \\
& f_{3}^{\prime}(x)=8 x \log x-4 x^{3}+6 x-4 x^{-1}+2 x^{-3}, \\
& f_{3}^{\prime}(1)=0, \\
& f_{3}^{\prime \prime}(x)=8 \log x-12 x^{2}+14+4 x^{-2}-6 x^{-4}, \\
& f_{3}^{\prime \prime}(1)=0
\end{aligned}
$$

and

$$
f_{3}^{\prime \prime \prime}(x)=-\frac{8}{x^{3}}(x+1)(x-1)\left(3 x^{4}+2 x^{2}+3\right)<0
$$

for $x>1$.

Inequality (2.11) implies that $f_{3}^{\prime \prime}(x)$ is strictly decreasing in $[1,+\infty)$, then equation (2.10) leads to the conclusion that $f_{3}^{\prime}(x)$ is strictly decreasing in $[1,+\infty)$.

From equations (2.4)-(2.9) and the monotonicity of $f_{3}^{\prime}(x)$, we clearly see that

$$
f_{1}(x)<0
$$

for $x>1$. 
Therefore, inequality (2.1) follows from equations (2.2) and (2.3) together with inequality (2.12).

Lemma 2.2 The inequality

$$
\left(\frac{x^{5}-1}{5 \log x}\right)^{1 / 5}<\frac{x-1}{2 \arctan \frac{x-1}{x+1}}
$$

holds for all $x>1$.

Proof Let

$$
g(x)=\frac{1}{5} \log \left(\frac{x^{5}-1}{5 \log x}\right)-\log \left(\frac{x-1}{2 \arctan \frac{x-1}{x+1}}\right) .
$$

Then simple computations lead to

$$
\begin{aligned}
& g(1)=0, \\
& g^{\prime}(x)=\frac{\left[5 x\left(x^{3}+x^{2}+x+1\right) \log x+x^{5}-1\right] g_{1}(x)}{5 x\left(x^{5}-1\right) \arctan \left(\frac{x-1}{x+1}\right) \log x},
\end{aligned}
$$

where

$$
\begin{aligned}
& g_{1}(x)=\frac{5 x\left(x^{5}-1\right) \log x}{\left(1+x^{2}\right)\left[5 x\left(x^{3}+x^{2}+x+1\right) \log x+x^{5}-1\right]}-\arctan \frac{x-1}{x+1}, \\
& g_{1}(1)=0, \\
& g_{1}^{\prime}(x)=\frac{x^{3} g_{2}(x)}{\left(1+x^{2}\right)^{2}\left[5 x\left(x^{3}+x^{2}+x+1\right) \log x+x^{5}-1\right]^{2}},
\end{aligned}
$$

where

$$
\begin{aligned}
g_{2}(x)= & 50\left(x^{6}+x^{5}-x^{4}+2 x^{3}-x^{2}+x+1\right) \log ^{2} x \\
& -5\left(x^{9}+2 x^{8}+x^{7}+4 x^{6}+4 x^{5}-4 x-4-x^{-1}-2 x^{-2}-x^{-3}\right) \log x \\
& +4\left(x^{9}+x^{7}-2 x^{4}-2 x^{2}+x^{-1}+x^{-3}\right), \\
g_{2}(1)= & 0 \\
g_{2}^{\prime}(x)= & 50\left(6 x^{5}+5 x^{4}-4 x^{3}+6 x^{2}-2 x+1\right) \log ^{2} x \\
& +5\left(-9 x^{8}-16 x^{7}-7 x^{6}-4 x^{5}-20 x^{3}+40 x^{2}-20 x+24+20 x^{-1}\right. \\
& \left.-x^{-2}-4 x^{-3}-3 x^{-4}\right) \log x+31 x^{8}-10 x^{7}+23 x^{6}-20 x^{5} \\
& -20 x^{4}-32 x^{3}-16 x+20+20 x^{-1}+x^{-2}+10 x^{-3}-7 x^{-4}, \\
g_{2}^{\prime}(1)= & 0, \\
g_{2}^{\prime \prime}(x)= & 100\left(15 x^{4}+10 x^{3}-6 x^{2}+6 x-1\right) \log ^{2} x+10\left(-36 x^{7}-56 x^{6}\right. \\
& -21 x^{5}+50 x^{4}+50 x^{3}-70 x^{2}+100 x-30+10 x^{-1}-10 x^{-2}+x^{-3} \\
& \left.+6 x^{-4}+6 x^{-5}\right) \log x+203 x^{7}-150 x^{6}+103 x^{5}-120 x^{4}-80 x^{3} \\
& -196 x^{2}+200 x-116+120 x^{-1}+80 x^{-2}-7 x^{-3}-50 x^{-4}+13 x^{-5},
\end{aligned}
$$




$$
\begin{aligned}
g_{2}^{\prime \prime}(1)= & 0 \\
g_{2}^{\prime \prime \prime}(x)= & 600\left(10 x^{3}+5 x^{2}-2 x+1\right) \log ^{2} x+10\left(-252 x^{6}-336 x^{5}-105 x^{4}\right. \\
& +500 x^{3}+350 x^{2}-260 x+220-20 x^{-1}-10 x^{-2}+20 x^{-3} \\
& \left.-3 x^{-4}-24 x^{-5}-30 x^{-6}\right) \log x+1,061 x^{6}-1,460 x^{5}+305 x^{4} \\
& +20 x^{3}+260 x^{2}-1,092 x+1,200-300 x^{-1}-20 x^{-2}-260 x^{-3} \\
& +31 x^{-4}+260 x^{-5}-5 x^{-6}, \\
g_{2}^{\prime \prime \prime}(1)= & 0 \\
g_{2}^{(4)}(x)= & 2 g_{3}(x),
\end{aligned}
$$

where

$$
\begin{aligned}
& g_{3}(x)=600\left(15 x^{2}+5 x-1\right) \log ^{2} x+10\left(-756 x^{5}-840 x^{4}\right. \\
& -210 x^{3}+1,300 x^{2}+650 x-250+6 x^{-1}+10 x^{-2}+10 x^{-3} \\
& \left.-30 x^{-4}+6 x^{-5}+60 x^{-6}+90 x^{-7}\right) \log x+1,923 x^{5}-5,330 x^{4} \\
& +85 x^{3}+2,530 x^{2}+2,010 x-1,846+1,100 x^{-1}+50 x^{-2}-30 x^{-3} \\
& +490 x^{-4}-77 x^{-5}-770 x^{-6}-135 x^{-7} \text {, } \\
& g_{3}(1)=0 \text {, } \\
& g_{3}^{\prime}(x)=3,000(6 x+1) \log ^{2} x+10\left(-3,780 x^{4}-3,360 x^{3}-630 x^{2}+4,400 x\right. \\
& +1,250-120 x^{-1}-6 x^{-2}-20 x^{-3}-30 x^{-4}+120 x^{-5}-30 x^{-6} \\
& \left.-360 x^{-7}-630 x^{-8}\right) \log x+2,055 x^{4}-29,720 x^{3}-1,845 x^{2}+18,060 x+8,510 \\
& -2,500 x^{-1}-1,040 x^{-2}+190 x^{-4}-2,260 x^{-5}+445 x^{-6}+5,220 x^{-7}+1,845 x^{-8} \\
& <3,000(6 x+1)(x-1) \log x+10\left(-3,780 x^{4}-3,360 x^{3}-630 x^{2}\right. \\
& +4,400 x+1,250-120 x^{-1}-6 x^{-2}-20 x^{-3}-30 x^{-4}+120 x^{-5}-30 x^{-6} \\
& \left.-360 x^{-7}-630 x^{-8}\right) \log x+2,055 x^{4}-29,720 x^{3}-1,845 x^{2}+18,060 x+8,510 \\
& -2,500 x^{-1}-1,040 x^{-2}+190 x^{-4}-2,260 x^{-5}+445 x^{-6}+5,220 x^{-7}+1,845 x^{-8} \\
& =10\left(-3,780 x^{4}-3,360 x^{3}+1,170 x^{2}+2,900 x+950-120 x^{-1}-6 x^{-2}\right. \\
& \left.-20 x^{-3}-30 x^{-4}+120 x^{-5}-30 x^{-6}-360 x^{-7}-630 x^{-8}\right) \log x \\
& +2,055 x^{4}-29,720 x^{3}-1,845 x^{2}+18,060 x+8,510-2,500 x^{-1}-1,040 x^{-2} \\
& +190 x^{-4}-2,260 x^{-5}+445 x^{-6}+5,220 x^{-7}+1,845 x^{-8} \text {. }
\end{aligned}
$$

Let

$$
\begin{aligned}
g_{4}(x)= & 10\left(-3,780 x^{4}-3,360 x^{3}+1,170 x^{2}+2,900 x+950-120 x^{-1}-6 x^{-2}\right. \\
& \left.-20 x^{-3}-30 x^{-4}+120 x^{-5}-30 x^{-6}-360 x^{-7}-630 x^{-8}\right) \log x \\
& +2,055 x^{4}-29,720 x^{3}-1,845 x^{2}+18,060 x+8,510-2,500 x^{-1} \\
& -1,040 x^{-2}+190 x^{-4}-2,260 x^{-5}+445 x^{-6}+5,220 x^{-7}+1,845 x^{-8} .
\end{aligned}
$$


Then

$$
\begin{aligned}
& g_{4}(1)=-1,040<0, \\
& g_{4}^{\prime}(x)=10 x^{-9} g_{5}(x),
\end{aligned}
$$

where

$$
\begin{aligned}
g_{5}(x)= & \left(-15,120 x^{12}-10,080 x^{11}+2,340 x^{10}+2,900 x^{9}+120 x^{7}+12 x^{6}\right. \\
& \left.+60 x^{5}+120 x^{4}-600 x^{3}+180 x^{2}+2,520 x+5,040\right) \log x-2,958 x^{12} \\
& -12,276 x^{11}+801 x^{10}+4,706 x^{9}+950 x^{8}+130 x^{7}+202 x^{6}-20 x^{5} \\
& -106 x^{4}+1,250 x^{3}-297 x^{2}-4,014 x-2,106 \\
< & \left(-15,120 x^{11}-10,080 x^{11}+2,340 x^{11}+2,900 x^{11}+120 x^{11}+12 x^{11}\right. \\
& \left.+60 x^{11}+120 x^{11}-600 x^{3}+180 x^{11}+2,520 x^{11}+5,040 x^{11}\right) \log x \\
& -2,958 x^{11}-12,276 x^{11}+801 x^{11}+4,706 x^{11}+950 x^{11}+130 x^{11} \\
& +202 x^{11}-20 x^{5}-106 x^{4}+1,250 x^{11}-297 x^{2}-4,014 x-2,106 \\
= & -\left(11,908 x^{11}+600 x^{3}\right) \log x-7,195 x^{11}-20 x^{5}-106 x^{4}-297 x^{2}-4,014 x-2,106 \\
< & 0
\end{aligned}
$$

for $x>1$.

Equation (2.27) and inequality (2.28) lead to the conclusion that $g_{4}(x)$ is strictly decreasing in $[1,+\infty)$. Then equation (2.26) implies that

$$
g_{4}(x)<0
$$

for $x>1$.

Inequalities (2.25) and (2.29) imply that $g_{3}^{\prime}(x)<0$. Then equation $(2.24)$ shows that

$$
g_{3}(x)<0
$$

for $x>1$.

From equations (2.17)-(2.23) and inequality (2.30), we clearly see that

$$
g_{1}(x)<0
$$

for $x>1$.

Therefore, inequality (2.13) follows easily from equations (2.14)-(2.16) and inequality (2.31).

\section{Lemma 2.3 The inequality}

$$
\operatorname{arcsinh}^{4}\left(\frac{x-1}{x+1}\right)-\frac{(x-1)^{4} \log x}{4\left(x^{4}-1\right)}<0
$$

holds for all $x>1$. 
Proof Let

$$
h(x)=\log \left[\operatorname{arcsinh}^{4}\left(\frac{x-1}{x+1}\right)\right]-\log \left[\frac{(x-1)^{4} \log x}{4\left(x^{4}-1\right)}\right] .
$$

Then simple computations lead to

$$
\begin{aligned}
& h(1)=0, \\
& h^{\prime}(x)=\frac{\left[4 x\left(x^{2}+x+1\right) \log x+x^{4}-1\right] h_{1}(x)}{x\left(x^{4}-1\right) \operatorname{arcsinh}\left(\frac{x-1}{x+1}\right) \log x},
\end{aligned}
$$

where

$$
\begin{aligned}
h_{1}(x) & =\frac{4 \sqrt{2} x\left(x^{4}-1\right) \log x}{(x+1)\left[4 x\left(x^{2}+x+1\right) \log x+x^{4}-1\right] \sqrt{1+x^{2}}} \\
& -\operatorname{arcsinh}\left(\frac{x-1}{x+1}\right), \\
h_{1}(1)= & 0, \\
h_{1}^{\prime}(x) & =\frac{\sqrt{2} x^{2} h_{2}(x)}{(x+1)\left(1+x^{2}\right)^{3 / 2}\left[4 x\left(x^{2}+x+1\right) \log x+x^{4}-1\right]^{2}},
\end{aligned}
$$

where

$$
\begin{aligned}
h_{2}(x)= & 16\left(x^{6}+x^{5}+2 x^{3}+x+1\right) \log ^{2} x-4\left(x^{4}-1\right)\left(x^{4}+x^{3}+3 x^{2}\right. \\
& \left.+2 x+3+x^{-1}+x^{-2}\right) \log x+3\left(x^{4}-1\right)^{2}\left(1+x^{-2}\right) \\
h_{2}(1)= & 0 \\
h_{2}^{\prime}(x)= & 2 h_{3}(x)
\end{aligned}
$$

where

$$
\begin{aligned}
h_{3}(x)= & 8\left(6 x^{5}+5 x^{4}+6 x^{2}+1\right) \log ^{2} x-2\left(8 x^{7}+7 x^{6}+10 x^{5}+2 x^{4}+8 x^{3}\right. \\
& \left.-16 x^{2}-4 x-10-8 x^{-1}+x^{-2}+2 x^{-3}\right) \log x+10 x^{7}-2 x^{6} \\
& +3 x^{5}-4 x^{4}-16 x^{3}-2 x+4+6 x^{-1}+2 x^{-2}-x^{-3}, \\
h_{3}(1)= & 0, \\
h_{3}^{\prime}(x)= & x h_{4}(x), \\
h_{4}(x)= & 16\left(15 x^{3}+10 x^{2}+6\right) \log ^{2} x-4\left(28 x^{5}+21 x^{4}+x^{3}-16 x^{2}+12 x\right. \\
& \left.-40-2 x^{-1}-4 x^{-2}+4 x^{-3}-x^{-4}-3 x^{-5}\right) \log x+54 x^{5}-26 x^{4} \\
& -5 x^{3}-20 x^{2}-64 x+32+6 x^{-1}+20 x^{-2}+10 x^{-3}-6 x^{-4}-x^{-5}, \\
h_{4}(1)= & 0, \\
h_{4}^{\prime}(x)= & x h_{5}(x),
\end{aligned}
$$


where

$$
\begin{aligned}
h_{5}(x)= & 80(9 x+4) \log ^{2} x-4\left(140 x^{3}+84 x^{2}-117 x-112+12 x^{-1}-48 x^{-2}\right. \\
& \left.+2 x^{-3}+8 x^{-4}-12 x^{-5}+4 x^{-6}+15 x^{-7}\right) \log x+158 x^{3}-188 x^{2}-19 x \\
& +24-112 x^{-1}+160 x^{-2}+2 x^{-3}-24 x^{-4}-46 x^{-5}+28 x^{-6}+17 x^{-7}, \\
h_{5}(1)= & 0 \\
h_{5}^{\prime}(x)= & 720 \log ^{2} x+4\left(-420 x^{2}-168 x+477+160 x^{-1}+12 x^{-2}-96 x^{-3}\right. \\
& \left.+6 x^{-4}+32 x^{-5}-60 x^{-6}+24 x^{-7}+105 x^{-8}\right) \log x-86 x^{2}-712 x \\
& +449+448 x^{-1}+64 x^{-2}-128 x^{-3}-14 x^{-4}+64 x^{-5}+278 x^{-6} \\
& -184 x^{-7}-179 x^{-8} \\
h_{5}^{\prime}(1)= & 0 \\
h_{5}^{\prime \prime}(x)= & 4 x^{-9} h_{6}(x),
\end{aligned}
$$

where

$$
\begin{aligned}
h_{6}(x)= & -8\left(105 x^{10}+21 x^{9}-45 x^{8}+20 x^{7}+3 x^{6}-36 x^{5}+3 x^{4}+20 x^{3}\right. \\
& \left.-45 x^{2}+21 x+105\right) \log x-\left(x^{2}-1\right)\left(463 x^{8}+346 x^{7}-14 x^{6}\right. \\
& \left.+298 x^{5}+6 x^{4}+298 x^{3}-14 x^{2}+346 x+463\right) \\
< & 0
\end{aligned}
$$

for all $x>1$.

Equations (2.45) and (2.46) together with inequality (2.47) imply that $h_{5}(x)$ is strictly decreasing in $[1,+\infty)$. Then equation (2.44) leads to

$$
h_{5}(x)<0
$$

for all $x>1$.

From equations (2.36)-(2.43) and inequality (2.48), we clearly see that

$$
h_{1}(x)<0
$$

for all $x>1$.

Therefore, inequality (2.32) follows from equations (2.33)-(2.35) and inequality (2.49).

\section{Main results}

Theorem 3.1 The inequality

$$
P(a, b)>L_{2}(a, b)
$$

holds for all $a, b>0$ with $a \neq b$, and $L_{2}(a, b)$ is the best possible lower generalized logarithmic mean bound for the first Seiffert mean $P(a, b)$. 
Proof From (1.2) and (1.4), we clearly see that both $P(a, b)$ and $L_{r}(a, b)$ are symmetric and homogenous of degree one. Without loss of generality, we assume that $b=1$ and $a=x^{2}>1$. Then (1.2) and (1.4) lead to

$$
L_{2}\left(x^{2}, 1\right)-P\left(x^{2}, 1\right)=\left(\frac{x^{4}-1}{4 \log x}\right)^{1 / 2}-\frac{x^{2}-1}{4 \arctan x-\pi} .
$$

Therefore, $P\left(x^{2}, 1\right)>L_{2}\left(x^{2}, 1\right)$ follows from Lemma 2.1 and equation (3.1).

Next, we prove that $L_{2}(a, b)$ is the best possible lower generalized logarithmic mean bound for the first Seiffert mean $P(a, b)$.

For any $\epsilon>0$ and $x>0$, from (1.2) and (1.4), one has

$$
L_{2+\epsilon}(1+x, 1)-P(1+x, 1)=\left[\frac{(1+x)^{2+\epsilon}-1}{(2+\epsilon) \log (1+x)}\right]^{1 /(2+\epsilon)}-\frac{x}{4 \arctan \sqrt{1+x}-\pi} .
$$

Letting $x \rightarrow 0$ and making use of Taylor expansion, we get

$$
\begin{aligned}
& {\left[\frac{(1+x)^{2+\epsilon}-1}{(2+\epsilon) \log (1+x)}\right]^{1 /(2+\epsilon)}-\frac{x}{4 \arctan \sqrt{1+x}-\pi}} \\
& \quad=\left[1+\frac{2+\epsilon}{2} x+\frac{(2+\epsilon)(1+2 \epsilon)}{12} x^{2}+o\left(x^{2}\right)\right]^{1 /(2+\epsilon)}-\frac{x}{x-\frac{1}{2} x^{2}+\frac{7}{24} x^{3}+o\left(x^{3}\right)} \\
& \quad=\left[1+\frac{1}{2} x-\frac{1-\epsilon}{24} x^{2}+o\left(x^{2}\right)\right]-\left[1+\frac{1}{2} x-\frac{1}{24} x^{2}+o\left(x^{2}\right)\right] \\
& =\frac{\epsilon}{24} x^{2}+o\left(x^{2}\right) .
\end{aligned}
$$

Equations (3.2) and (3.3) imply that for any $\epsilon>0$, there exists $\delta_{1}=\delta_{1}(\epsilon)>0$ such that $L_{2+\epsilon}(1+x, 1)>P(1+x, 1)$ for $x \in\left(0, \delta_{1}\right)$.

Remark 3.1 It follows from (1.2) and (1.4) that

$$
\lim _{x \rightarrow+\infty} \frac{P(x, 1)}{L_{\lambda}(x, 1)}=\lim _{x \rightarrow+\infty} \frac{\lambda^{1 / \lambda}(1-1 / x) \log ^{1 / \lambda} x}{\pi\left(1-x^{-\lambda}\right)^{1 / \lambda}}=+\infty
$$

for all $\lambda>0$.

Equation (3.4) implies that $\lambda>0$ such that $L_{\lambda}(a, b)>P(a, b)$ for all $a, b>0$ does not exist.

Theorem 3.2 The inequality

$$
T(a, b)>L_{5}(a, b)
$$

holds for all $a, b>0$ with $a \neq b$, and $L_{5}(a, b)$ is the best possible lower generalized logarithmic mean bound for the second Seiffert mean $T(a, b)$.

Proof Without loss of generality, we assume that $b=1$ and $a=x>1$. Then (1.3) and (1.4) lead to

$$
L_{5}(x, 1)-T(x, 1)=\left(\frac{x^{5}-1}{5 \log x}\right)^{1 / 5}-\frac{x-1}{2 \arctan \frac{x-1}{x+1}} .
$$


Therefore, $T(x, 1)>L_{5}(x, 1)$ follows from Lemma 2.2 and equation (3.5).

Next, we prove that $L_{5}(a, b)$ is the best possible lower generalized logarithmic mean bound for the second Seiffert mean $T(a, b)$.

For any $\epsilon>0$ and $x>0$, from (1.3) and (1.4), one has

$$
L_{5+\epsilon}(1+x, 1)-T(1+x, 1)=\left[\frac{(1+x)^{5+\epsilon}-1}{(5+\epsilon) \log (1+x)}\right]^{1 /(5+\epsilon)}-\frac{x}{2 \arctan \frac{x}{2+x}} .
$$

Letting $x \rightarrow 0$ and making use of Taylor expansion, we have

$$
\begin{aligned}
& {\left[\frac{(1+x)^{5+\epsilon}-1}{(5+\epsilon) \log (1+x)}\right]^{1 /(5+\epsilon)}-\frac{x}{2 \arctan \frac{x}{2+x}}} \\
& \quad=\left[1+\frac{5+\epsilon}{2} x+\frac{(5+\epsilon)(7+2 \epsilon)}{12} x^{2}+o\left(x^{2}\right)\right]^{1 /(5+\epsilon)}-\frac{x}{x-\frac{1}{2} x^{2}+\frac{1}{6} x^{3}+o\left(x^{3}\right)} \\
& \quad=\left[1+\frac{1}{2} x+\frac{2+\epsilon}{24} x^{2}+o\left(x^{2}\right)\right]-\left[1+\frac{1}{2} x+\frac{1}{12} x^{2}+o\left(x^{2}\right)\right] \\
& \quad=\frac{\epsilon}{24} x^{2}+o\left(x^{2}\right) .
\end{aligned}
$$

Equations (3.6) and (3.7) imply that for any $\epsilon>0$, there exists $\delta_{2}=\delta_{2}(\epsilon)>0$ such that $L_{5+\epsilon}(1+x, 1)>T(1+x, 1)$ for $x \in\left(0, \delta_{2}\right)$.

Remark 3.2 It follows from (1.3) and (1.4) that

$$
\lim _{x \rightarrow+\infty} \frac{T(x, 1)}{L_{\mu}(x, 1)}=\lim _{x \rightarrow+\infty} \frac{2 \mu^{1 / \mu}(1-1 / x) \log ^{1 / \mu} x}{\pi\left(1-x^{-\mu}\right)^{1 / \mu}}=+\infty
$$

for all $\mu>0$.

Equation (3.8) implies that $\mu>0$ such that $L_{\mu}(a, b)>T(a, b)$ for all $a, b>0$ does not exist.

Theorem 3.3 The inequality

$$
M(a, b)>L_{4}(a, b)
$$

holds for all $a, b>0$ with $a \neq b$, and $L_{4}(a, b)$ is the best possible lower generalized logarithmic mean bound for the Neuman-Sándor mean $M(a, b)$.

Proof Without loss of generality, we assume that $b=1$ and $a=x>1$. Then (1.1) and (1.4) lead to

$$
\begin{aligned}
& L_{4}^{4}(x, 1)-M^{4}(x, 1) \\
& \quad=\frac{x^{4}-1}{4 \log x}-\frac{(x-1)^{4}}{16 \operatorname{arcsinh}^{4}\left(\frac{x-1}{x+1}\right)} \\
& \quad=\frac{x^{4}-1}{4 \operatorname{arcsinh}^{4}\left(\frac{x-1}{x+1}\right) \log x}\left[\operatorname{arcsinh}^{4}\left(\frac{x-1}{x+1}\right)-\frac{(x-1)^{4} \log x}{4\left(x^{4}-1\right)}\right] .
\end{aligned}
$$

Therefore, $M(x, 1)>L_{4}(x, 1)$ follows from Lemma 2.3 and equation (3.9). 
Next, we prove that $L_{4}(a, b)$ is the best possible lower generalized logarithmic mean bound for the Neuman-Sándor mean $M(a, b)$.

For any $\epsilon>0$ and $x>0$, from (1.1) and (1.4), one has

$$
L_{4+\epsilon}(1+x, 1)-T(1+x, 1)=\left[\frac{(1+x)^{4+\epsilon}-1}{(4+\epsilon) \log (1+x)}\right]^{1 /(4+\epsilon)}-\frac{x}{2 \operatorname{arcsinh}\left(\frac{x}{2+x}\right)} .
$$

Letting $x \rightarrow 0$ and making use of Taylor expansion, we have

$$
\begin{aligned}
& {\left[\frac{(1+x)^{4+\epsilon}-1}{(4+\epsilon) \log (1+x)}\right]^{1 /(4+\epsilon)}-\frac{x}{2 \operatorname{arcsinh}\left(\frac{x}{2+x}\right)}} \\
& \quad=\left[1+\frac{4+\epsilon}{2} x+\frac{(4+\epsilon)(5+2 \epsilon)}{12} x^{2}+o\left(x^{2}\right)\right]^{1 /(4+\epsilon)}-\frac{x}{x-\frac{1}{2} x^{2}+\frac{5}{24} x^{3}+o\left(x^{3}\right)} \\
& \quad=\left[1+\frac{1}{2} x+\frac{1+\epsilon}{24} x^{2}+o\left(x^{2}\right)\right]-\left[1+\frac{1}{2} x+\frac{1}{24} x^{2}+o\left(x^{2}\right)\right] \\
& \quad=\frac{\epsilon}{24} x^{2}+o\left(x^{2}\right) .
\end{aligned}
$$

Equations (3.10) and (3.11) imply that for any $\epsilon>0$, there exists $\delta_{3}=\delta_{3}(\epsilon)>0$ such that $L_{4+\epsilon}(1+x, 1)>M(1+x, 1)$ for $x \in\left(0, \delta_{3}\right)$.

Remark 3.3 It follows from (1.1) and (1.4) that

$$
\lim _{x \rightarrow+\infty} \frac{M(x, 1)}{L_{v}(x, 1)}=\lim _{x \rightarrow+\infty} \frac{v^{1 / v}(1-1 / x) \log ^{1 / v} x}{2 \operatorname{arcsinh}(1)\left(1-x^{-\nu}\right)^{1 / v}}=+\infty
$$

for all $v>0$.

Equation (3.12) implies that $v>0$ such that $L_{v}(a, b)>M(a, b)$ for all $a, b>0$ does not exist.

\section{Competing interests}

The authors declare that they have no competing interests.

\section{Authors' contributions}

YMC provided the main idea and carried out the proof of Remarks 3.1-3.3 in this article. BYL carried out the proof of Lemma 2.1 and Theorem 3.1 in this article. WMG carried out the proof of Lemma 2.2 and Theorem 3.2 in this article. YQS carried out the proof of Lemma 2.3 and Theorem 3.3 in this article. All authors read and approved the final manuscript.

\section{Author details}

${ }^{1}$ School of Mathematics and Computation Sciences, Hunan City University, Yiyang, 413000, China. ${ }^{2}$ College of Mathematics Science, Anhui University, Hefei, 230039, China.

\section{Acknowledgements}

This research was supported by the Natural Science Foundation of China under Grants 11071069 and 11171307, the Natural Science Foundation of Hunan Province under Grant 09JJ6003 and the Innovation Team Foundation of the Department of Education of Zhejiang Province under Grant T200924.

Received: 15 June 2012 Accepted: 18 December 2012 Published: 7 January 2013

\section{References}

1. Neuman, E, Sándor, J: On the Schwab-Borchardt mean. Math. Pannon. 14(2), 253-266 (2003)

2. Seiffert, H-J: Problem 887. Nieuw Arch. Wiskd. 11, 176 (1993)

3. Seiffert, H-J: Aufgabe $\beta$ 16. Die Wurzel 29, 221-222 (1995)

4. Chu, Y-M, Qiu, Y-F, Wang, M-K: Sharp power mean bounds for combination of Seiffert and geometric means. Abstr. Appl. Anal. 2010, Article ID 108920 (2010) 
5. Chu, Y-M, Qiu, Y-F, Wang, M-K, Wang, G-D: The optimal convex combination bounds of arithmetic and harmonic means for the Seiffert's means. J. Inequal. Appl. 2010, Article ID 436457 (2010)

6. Liu, H, Meng, X-J: The optimal convex combination bounds for the Seiffert's mean. J. Inequal. Appl. 2011, Article ID $686834(2011)$

7. Sándor, J: On certain inequalities for means III. Arch. Math. 76(1), 30-40 (2001)

8. Seiffert, H-J: Ungleichunen für einen bestimmten Mittelwert. Nieuw Arch. Wiskd. 13(2), 195-198 (1995)

9. Bullen, PS, Mitrinović, DS, Vasić, PM: Means and Their Inequalities. Reidel, Dordrecht (1988)

10. Jagers, AA: Solution of problem 887. Nieuw Arch. Wiskd. 12, 230-231 (1994)

11. Hästö, PA: A monotonicity property of ratios of symmetric homogeneous means. J. Inequal. Pure Appl. Math. 3(5), Article 71 (2002)

12. Hästö, PA: Optimal inequalities between Seiffert's mean and power mean. Math. Inequal. Appl. 7(1), 47-53 (2004)

13. Wang, M-K, Qiu, Y-F, Chu, Y-M: Sharp bounds for Seiffert means in terms of Lehmer means. J. Math. Inequal. 4(4), 581-586 (2010)

14. Chu, Y-M, Wang, M-K, Gong, W-M: Two sharp double inequalities for Seiffert mean. J. Inequal. Appl. 2010, 44 (2010)

15. Chu, Y-M, Zong, C, Wang, G-D: Optimal convex combination bounds of Seiffert and geometric means for the arithmetic mean. J. Math. Inequal. 5(3), 429-434 (2011)

16. Borwein, JM, Borwein, PB: Inequalities for compound mean iterations with logarithmic asymptotes. J. Math. Anal. Appl. 177(2), 572-582 (1993)

17. Vamanamurthy, MK, Vuorinen, M: Inequalities for means. J. Math. Anal. Appl. 183(1), 155-166 (1994)

doi:10.1186/1029-242X-2013-10

Cite this article as: Chu et al.: Sharp bounds for Seiffert and Neuman-Sándor means in terms of generalized logarithmic means. Journal of Inequalities and Applications 2013 2013:10.

\section{Submit your manuscript to a SpringerOpen ${ }^{\ominus}$ journal and benefit from:}

- Convenient online submission

- Rigorous peer review

- Immediate publication on acceptance

- Open access: articles freely available online

- High visibility within the field

- Retaining the copyright to your article 